\title{
BMJ Open Vaccination against COVID-19 and society's return to normality in England: a modelling study of impacts of different types of naturally acquired and vaccine-induced immunity
}

Fujian Song (), Max O Bachmann ()

To cite: Song F, Bachmann MO. Vaccination against COVID-19 and society's return to normality in England: a modelling study of impacts of different types of naturally acquired and vaccineinduced immunity. BMJ Open 2021;11:e053507. doi:10.1136/ bmjopen-2021-053507

- Prepublication history and additional supplemental material for this paper are available online. To view these files, please visit the journal online (http://dx.doi.org/10.1136/ bmjopen-2021-053507).

Received 17 May 2021 Accepted 19 October 2021

A) Check for updates

(c) Author(s) (or their employer(s)) 2021. Re-use permitted under CC BY-NC. No commercial re-use. See rights and permissions. Published by BMJ.

Norwich Medical School, University of East Anglia, Norwich, UK

Correspondence to

Dr Fujian Song;

fujian.song@uea.ac.uk

\section{ABSTRACT}

Objectives To project impacts of mass vaccination against COVID-19, and investigate possible impacts of different types of naturally acquired and vaccine-induced immunity on future dynamics of SARS-CoV-2 transmission from 2021 to 2024 in England.

Design Deterministic, compartmental, discrete-time Susceptible-Exposed-Infectious-Recovered (SEIR) modelling.

Participants Population in England.

Interventions Mass vaccination programmes.

Outcome measures Daily and cumulative number of deaths from COVID-19.

Results If vaccine efficacy remains high (85\%), the vaccine-induced sterilising immunity lasts $\geq 182$ days, and the reinfectivity is greatly reduced (by $\geq 60 \%$ ), annual mass vaccination programmes can prevent further COVID-19 outbreaks in England. Under optimistic scenarios, with annual revaccination programmes, the cumulative number of COVID-19 deaths is estimated to be from 130000 to 150000 by the end of 2024 . However, the total number of COVID-19 deaths may be up to 431000 by the end of 2024 , under scenarios with compromised vaccine efficacy $(62.5 \%)$, short duration of natural and vaccine immunity (365/182 days) and small reduction in reinfectivity (30\%). Under the assumed scenarios, more frequent revaccinations are associated with smaller total numbers and lower peaks of daily deaths from COVID-19.

Conclusions Under optimistic scenarios, mass immunisation using efficacious vaccines may enable society safely to return to normality. However, under plausible scenarios with low vaccine efficacy and short durability of immunity, COVID-19 could continue to cause recurrent waves of severe morbidity and mortality despite frequent vaccinations. It is crucial to monitor the vaccination effects in the real world, and to better understand characteristics of naturally acquired and vaccine-induced immunity against SARS-CoV-2.

\section{INTRODUCTION}

The COVID-19 pandemic caused by the spread of SARS-CoV-2 has resulted in more than 4.2 million deaths globally by the end of July 2021, ${ }^{1}$ and severe disruptions of
Strengths and limitations of this study

- This is the first modelling study to explicitly investigate the impacts of different types of immune responses to SARS-CoV-2 infection and vaccines on the COVID-19 epidemic in England.

- The model has been calibrated based on historically observed data in England, and plausible futures are explored via a large number of projection scenarios.

- This is a deterministic model to answer 'what-if' questions, and uncertainty in estimated parameters may have not been fully accommodated.

- There remain many uncertainties regarding durability and types of naturally acquired and vaccineinduced immunity against SARS-CoV-2.

economies and social activities around the world. The spread of the SARS-CoV-2 can be suppressed by non-pharmaceutical interventions (NPIs) and lockdown measures. ${ }^{2}$ Because of their disruptive socioeconomic consequences, lockdown restrictions cannot last indefinitely.

Only a few months after the initial identification of SARS-CoV-2 pathogen, there were more than 200 vaccine candidates in development globally. ${ }^{3}$ Results of randomised controlled trials showed that vaccines were efficacious in reducing symptomatic diseases, compared with placebo. ${ }^{45}$ Between 3 December 2020 and 31 July 2021, four vaccines against COVID-19 were approved for emergency use in the UK, and a vaccination programme has been started to roll out since 8 December 2020, prioritised primarily by age and comorbidity, with older people being vaccinated first. ${ }^{6}$ By 30 July 2021, the coverage rate of vaccination in adults aged $\geq 18$ in the UK was $88.5 \%$ for dose 1 and $72.1 \%$ for dose 2 of COVID-19 vaccines. ${ }^{7}$ Although the number of people infected with SARS-CoV-2 
in the UK was large during June to July 2021, numbers of hospitalised patients with COVID-19 and related deaths had remained relatively low, partly due to the protection of vaccines. For example, there were more than 56000 cases tested positive reported on 15 July 2021, while daily hospital admissions of patients with COVID-19 ranged from 593 to 836 during 15-31 July 2021, and the reported daily deaths due to COVID-19 ranged from 39 to 92 during the same period in England. ${ }^{7}$ Therefore, lockdown restrictions in England have been mostly lifted since 19 July 2021. ${ }^{8}$ Although mass vaccination is a promising strategy to enable society to safely return to normality, without mandatory NPIs, there is great uncertainty about the effects of COVID-19 vaccines and society's return to normality, including vaccines' long-term efficacy and emerging new variants of SARS-CoV-2. ${ }^{9} 10$

There have been many mathematical modelling studies to investigate COVID-19 dynamics and the impacts of control measures (eg, ref 11-13). Several modelling studies evaluated impacts of vaccines on the dynamics and consequences of COVID-19 epidemics in the UK. ${ }^{14-17}$ Two of these studies assessed impacts of the relaxation of social restriction after vaccination in the UK, ${ }^{15} 16$ and one study assessed impacts of vaccination on hospital admissions, ${ }^{17}$ but covered a shorter time horizon and did not consider waning of immunity. Another study of SARS-CoV-2 vaccination in the UK focused on economic evaluations. ${ }^{17}$ There have been some published modelling studies of vaccination against COVID-19 in other countries. For example, a modelling study examined the nationwide vaccination and returning to normal life in the USA, finding that vaccination alone was insufficient and NPI measures were still required. ${ }^{18}$

The protection of naturally acquired or vaccine-induced immune responses may be attributable to infection protection, disease reduction and reinfectivity reduction. ${ }^{19}$ Studies of diseases caused by other human coronaviruses (HCoVs) indicated that infection protection immunity is likely to be short lived, while disease reduction and reinfectivity reduction are likely long lasting. ${ }^{20}$ We are not aware of modelling studies that considered these important immunity characteristics in the evaluation of national mass vaccination programmes against COVID-19 epidemics. Therefore, we conducted a modelling study to investigate possible impacts of different types of naturally acquired and vaccine-induced immunity on future dynamics of SARS-CoV-2 transmission in England.

\section{METHODS}

\section{Model structure}

This is a deterministic, compartmental, discrete-time (day) population dynamic model, implemented with computational language $\mathrm{R}^{21}$ The population are classified into categories by sex, age ( 5 years age band for age $<10$ years, and 10 years age band for age $\geq 10$ years) and COVID-19 infection status (figure 1). The main infection compartments include susceptible, exposed, infectious, recovered and vaccinated. Here, 'exposed' refers to a preinfectious status of infected individuals. Infected individuals are classified as asymptomatic or symptomatic, and symptomatic individuals are classified

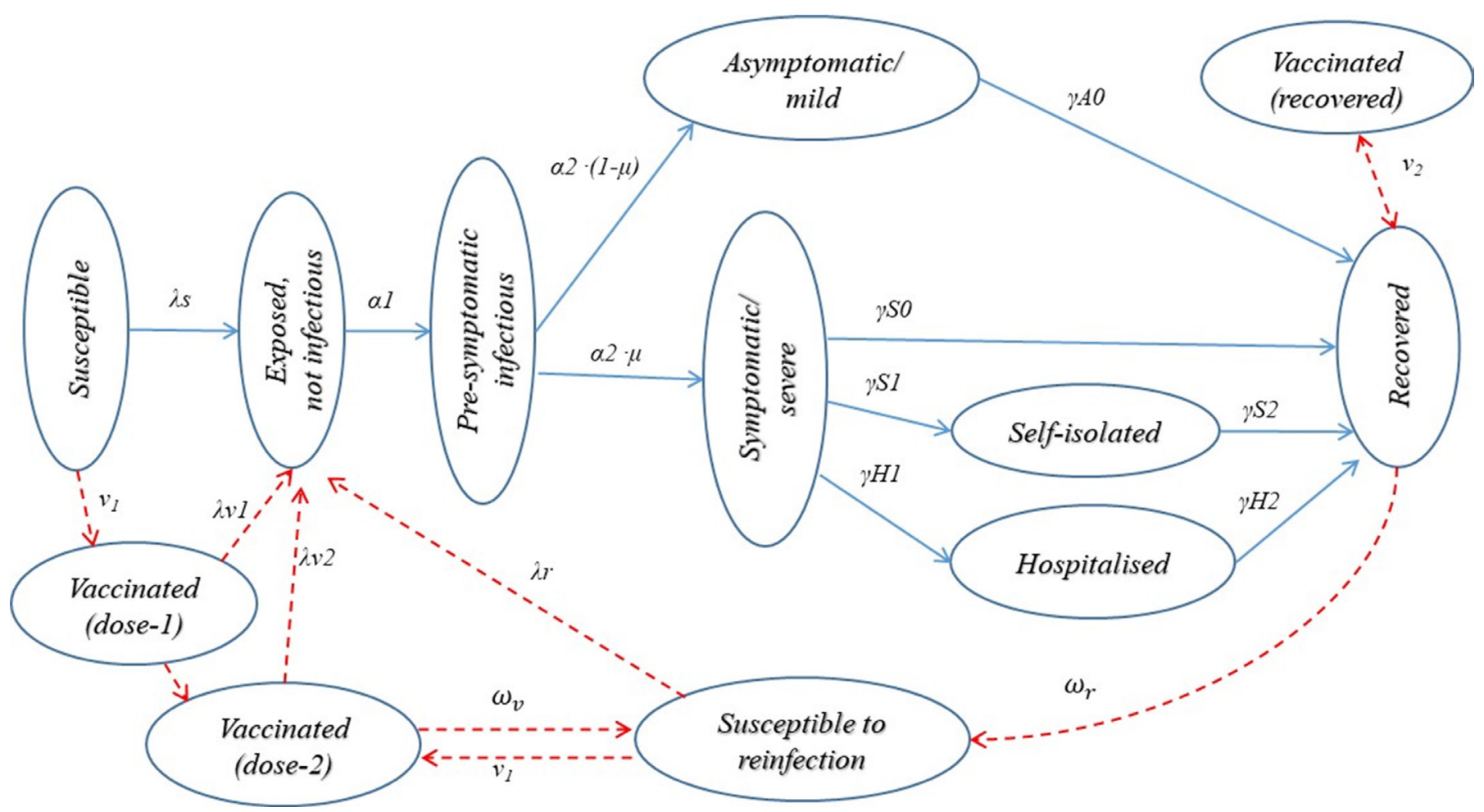

Figure 1 Modelling COVID-19 epidemics in England-main compartments and transitions across status. See online supplemental files for details on definitions of transition parameters. 
as not being isolated, self-isolated and hospitalised. We assume that hospitalised patients are effectively isolated and no longer able to transmit the virus to the general population, but patients who self-isolate at home may transmit virus to household contacts. The recovered and effectively vaccinated are protected from reinfection, but they may be reinfected if the immunity is short lived.

\section{Parameters and data sources}

Details on the model's structure (online supplemental appendix figure 1), parameters, data sources (online supplemental appendix table 1) and mathematical equations are available in online supplemental files. Initial parameter values were estimated based on a review of relevant literature, and key parameter estimates were calibrated so that the simulated numbers of COVID-19 deaths, hospitalised patients and infected individuals were as similar as possible to historically reported data from March 2020 to June 2021 in England. ${ }^{22}$

We obtained population demographic statistics in England from the Office for National Statistics, ${ }^{23}$ and the whole population is assumed to be susceptible to SARSCoV-2 infection at the beginning of 2020. We assume incubation periods, infectious periods, days of hospital stay and days of deaths after being infected to be gamma distributed (online supplemental appendix table 1). ${ }^{112425}$ Age-specific case fatality rates and hospitalisation rates of symptomatic cases were based on a study by Verity et $a l^{25}$ Average sex and age-specific rates of all-cause deaths from 2015 to 2019 in England $^{23}$ are applied to people who are not infected with or recovered from COVID-19. We assumed that the number of births equals to the number of deaths each day, and did not consider the influence of population migration. We adjusted the number of individuals belonging to each age group at the beginning of a year since 2021 by shifting a proportion of them to the adjacent higher age group.

Effects of NPI measures, including restrictions on social activities, contact tracing and testing, were materialised by changes in transmission risk per contact between a susceptible and an infectious individual, ${ }^{26}$ and average numbers of contacts of the general population (online supplemental appendix table 1). We estimate that the transmission risk per contact between infectious and susceptible individuals was reduced by $30 \%$, from 0.094 before the implementation of any NPIs to 0.066 by 15 March 2020 after implementing basic NPI measures. Because of the new virus variants, ${ }^{27}$ the average transmission risk per contact was increased to 0.077 by the end of 2020, and 0.081 since June 2021. We assume that, since September 2021, the transmission risk per contact is $10 \%$ higher in September, October, March and April, and 20\% higher in the winter months of November, December, January and February, compared with the risk in summer months from May to August. ${ }^{28}$ We do not use the reproduction number as an input parameter, but derived the basic and effective reproduction numbers based on model's transmission parameters (equation 55 in online supplemental files). ${ }^{1326}$

The sex and age-specific numbers of daily contacts per person were based on the UK data from a study of European countries. ${ }^{29}$ We consider only the daily contacts of the general population and household contacts of individuals self-isolated at home. We estimated that the lockdown measures from 24 March 2020 reduced general population contacts by $60 \%-85 \%$, although household contacts were unchanged. The NPI measures were relaxed or strengthened over time, which were reflected in the assumed social contacts and transmission risk (online supplemental files). Social contacts in England since 19 July 2021 are returned to normal as before the pandemic in England, although some basic hygienic measures would be maintained.

\section{Vaccination and projection scenarios}

Vaccination of prioritised individuals began on 8 December 2020 in England. ${ }^{30}$ The Joint Committee on Vaccination and Immunisation in the UK previously recommended COVID-19 vaccination of individuals aged $\geq 18$, and also recommended vaccination of young people aged 16-17 years on 4 August $2021 .{ }^{31}$ The vaccine uptake rate in adults aged $\geq 18$ was $88.5 \%$ for dose 1 and $72.1 \%$ for dose 2 by 30 July $2021,{ }^{22}$ and the coverage with two doses was $>90 \%$ in adults aged $\geq 65,80 \%-90 \%$ in those aged 50-64 and around $60 \%$ in those aged $40-49 .{ }^{32}$ In this study, the mass vaccination is modelled as an agebased phase approach, starting from people aged $\geq 70$, followed by individuals aged 60-69, 50-59, 30-49, and then those aged 16-29 years old. We assume that the mass vaccination starts from 1 January 2021, and the maximum number of individuals vaccinated daily is 180000 , to match with numbers of vaccinated individuals according to the official statistics. In the main analysis, we assume that the uptake rate is $75 \%, 80 \%, 85 \%$ and $90 \%$, respectively, in adults aged $16-29,30-39,40-49$ and $\geq 50$ years. Because of uncertain coverage of vaccination in younger people, we conducted sensitivity analyses under scenarios with lower coverage $(60 \%, 70 \%$ and $80 \%)$ and higher coverage $(80 \%, 85 \%$ and $90 \%)$ of vaccination in people aged 16-29, 30-39 and 40-49 years, respectively.

Both Pfizer-BioNTech and AstraZeneca vaccines are two-dose regimens, and the policy in the UK has been to initially provide the first dose to as many adults as possible. Data from clinical trials indicated that the short-term vaccine efficacy after the first dose of PfizerBioNTech and AstraZeneca vaccines is about $90 \%$ and $70 \%$, respectively. ${ }^{4}$ Public Health England (PHE) in July 2021 estimated that the efficacy was $55 \%-70 \%$ after the first dose, and $70 \%-85 \%$ or $85 \%-95 \%$ after the second dose. ${ }^{32}$ In this study, we assume that the overall vaccine efficacy is $62.5 \%$ after the first dose and $85 \%$ after the second dose. The protection effects start 14 days after the first dose vaccination, and the interval between the first and second doses is 9 weeks. The overall vaccine efficacy after the second dose may be lower than $85.0 \%$ due 
to new variants of SARS-CoV-2. Therefore, we assume a scenario in which the vaccine efficacy is reduced by new viral variants to be $44.0 \%$ after the first dose and $62.5 \%$ after the second dose during 2022-2024.

The reduction in symptomatic cases in vaccinated individuals may be due to induced antibodies in susceptible individuals (infection protection), or a reduction in symptomatic cases among infected individuals (disease reduction), or a combination of both. There are many different possible combinations of infection protection and disease reduction for a given overall vaccine efficacy in reducing symptomatic cases (online supplemental appendix figure 2 ). We assume that vaccine efficacy for reducing symptomatic cases is equally attributable to infection reduction and disease reduction in the main projections. For vaccines with $62.5 \%$ and $85.0 \%$ overall efficacy, the equal partial efficacy for the infection protection and for disease reduction is $38.8 \%$ and $61.3 \%$, respectively (see online supplemental files for details).

Immune responses against COVID-19 infection, either naturally acquired from prior infection or vaccine induced, may reduce individuals' susceptibility to infection (infection protection or sterilising immunity), reduce pathology so that disease is less severe after being infected (disease reduction immunity) and reduce infectivity of those who are reinfected after the waning of immunity (reinfectivity reduction immunity). ${ }^{19}$ According to immunological characteristics of other $\mathrm{HCoVs}$, infection protection immunity may wane after a short period, while disease protection and reinfectivity reduction immunity are likely longer lasting. ${ }^{20}$ For example, antibodies against SARSCoV-1 in recovered patients were no longer detectable after 2-3 years, while specific memory $\mathrm{T}$ cells remained detected after 11 years. ${ }^{33}$ Therefore, we assume that the disease reduction and reinfectivity reduction immunity are long lasting ( $>4$ years).$^{20}$ We assume that naturally acquired sterilising immunity lasts for 365 or 730 days, and vaccine-induced sterilising immunity lasts for 182 or 365 days. Available evidence indicated that the viral loads and the duration of virus shedding in the infected individuals after vaccination were considerably reduced, compared with unvaccinated individuals. ${ }^{34}$ PHE in July 2021 estimated that the reinfectivity was reduced by $35 \%-50 \%$ after the first dose of vaccines, ${ }^{32}$ although it is possible that the reduction in reinfectivity may be larger than $35 \%-50 \%$ after the second dose of vaccines. More recent studies reported that fully vaccinated individuals who were infected were between $41 \%$ and $78 \%$ less likely to transmit the virus to unvaccinated individuals. ${ }^{36}$ In this study, we assume that reinfectivity after waning of sterilising immunity is reduced by $30 \%, 45 \%$ or $60 \%$. We also assume that the infectivity of ineffectively vaccinated individuals is the same as recovered individuals whose sterilising immunity has waned, and vaccination of individuals recovered from natural infection boosts their naturally acquired immunity.

We considered scenarios with different frequency of revaccination programmes, including a single vaccination programme, multiple (2-4) annual revaccinations and revaccination programmes with different frequency and time intervals. The main characteristics of the simulated scenarios are summarised in online supplemental tables $1-3$.

We run the model and calibrate key transmission parameters by visually comparing estimated numbers of daily COVID-19 deaths, and hospitalised patients, with official records from 1 January 2020 to 30 June 2021 in England. ${ }^{22}$ We used estimates of transmission parameters by the end of June 2021 to project COVID-19 deaths from 2021 to 2024, under various scenarios of vaccine efficacy, durability and protection characteristics of naturally acquired and vaccine-induced immunity. The number of deaths from SARS-CoV-2 infections is the main endpoint in this study.

\section{Patient and public involvement}

No patients and the public were involved in this literature and secondary data-based, computational modelling study.

\section{RESULTS}

Our derived basic reproduction number (R0) was 3.68 at the initial stage of the COVID-19 epidemic in England. After implementing NPI and lockdown measures, the effective reproduction value (Rt) was reduced to 0.66 after 24 March 2020. Thereafter, the Rt values fluctuated along with changing NPI policies, and our estimated $\mathrm{R}$ values were within the ranges reported in England (online supplemental appendix figure 3). ${ }^{37}$ The estimated prevalence of SARS-CoV-2 infection (online supplemental appendix figure 4), the number of hospitalised patients with COVID-19 (online supplemental appendix figure 5) and the estimated daily deaths from COVID-19 (online supplemental appendix figure 6) are well matched with the reported data from March 2020 to June 2021 in England (online supplemental files).

\section{Vaccine efficacy, immunity durability and reinfectivity}

Figure 2 shows the impacts of partial vaccine efficacy regarding disease reduction relative to infection protection, durability of immunity and reinfectivity, given the same overall vaccine efficacy in reducing symptomatic cases. There are three general inferences. As expected, the number of COVID-19 deaths is smaller following a greater reduction in reinfectivity (figure $2 \mathrm{~A}-\mathrm{C}$ ). Second, a greater reduction in reinfectivity makes the durability of immunity less influential, if a vaccine is efficacious for infection protection (figure 2C). Third, the impacts of partial efficacy of infection protection relative to disease reduction by a vaccine are substantial when the duration of immunity is short lasting. A combination of a shorter duration of immunity and smaller reduction in the reinfectivity makes the disease reduction efficacy more beneficial (figure 2A,B). 


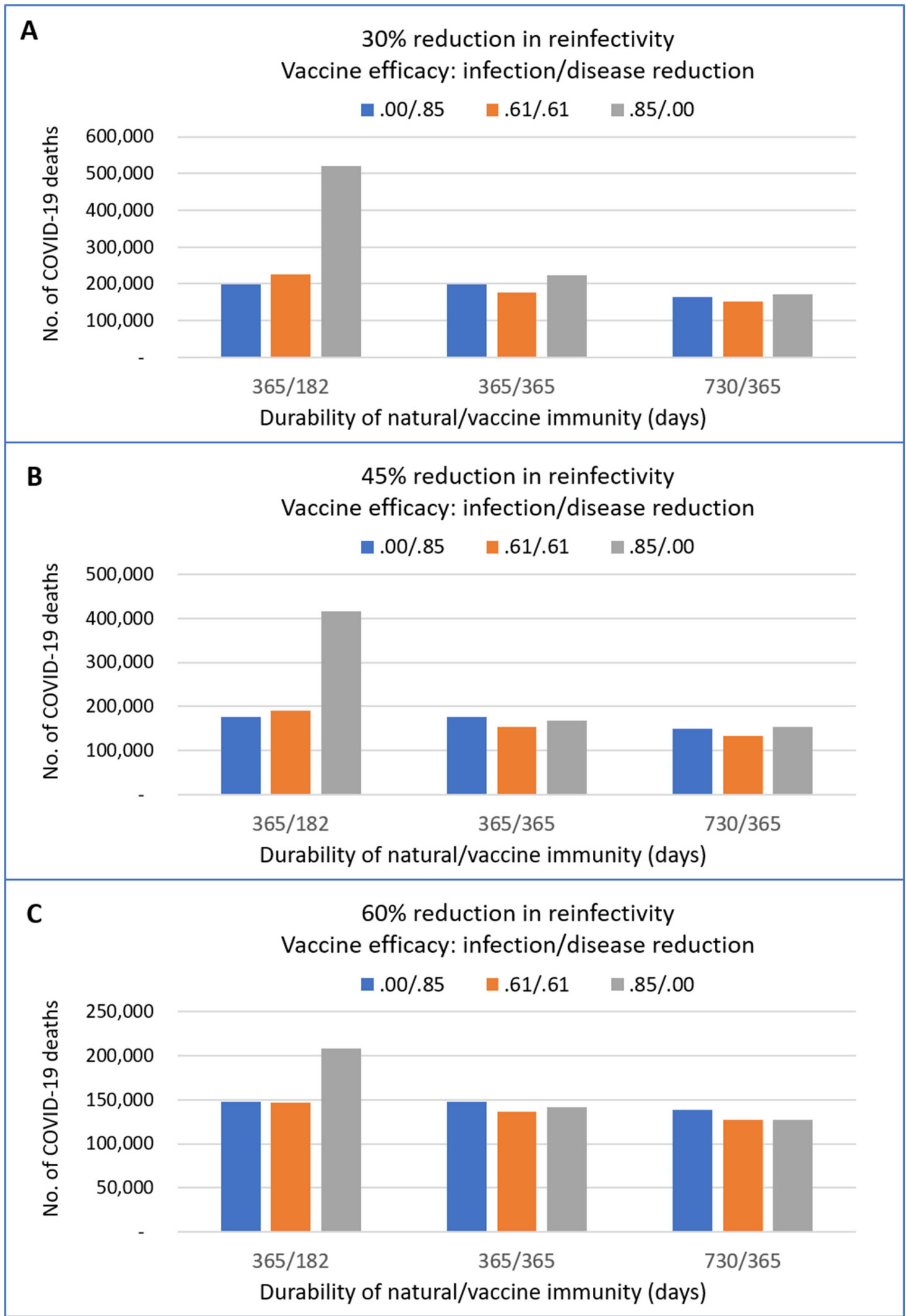

Figure 2 Projected numbers of COVID-19 deaths with different combinations of partial efficacy of infection and disease protection, immunity duration and reduction in reinfectivity. The cumulative number of COVID-19 deaths by the end of 2024, after four repeated annual vaccinations of $90 \%$ individuals aged $\geq 18$ during $2021-2024$. The overall vaccine efficacy was $85 \%$; ' $0.00 / 0.85$ ' refers to all vaccine efficacy attributable to disease protection, ' $0.61 / 0.61$ ' refers to equal infection and disease protection, '0.85/0.00' refers to all efficacy attributable to infection protection. Immunity duration: '365/182' refers to 365 and 182 days, and ' $730 / 365$ ' refers to 730 and 365 days, respectively, natural and vaccine immunity. A-C show results under the assumption of $30 \%, 45 \%$ and $60 \%$ reductions in reinfectivity. 


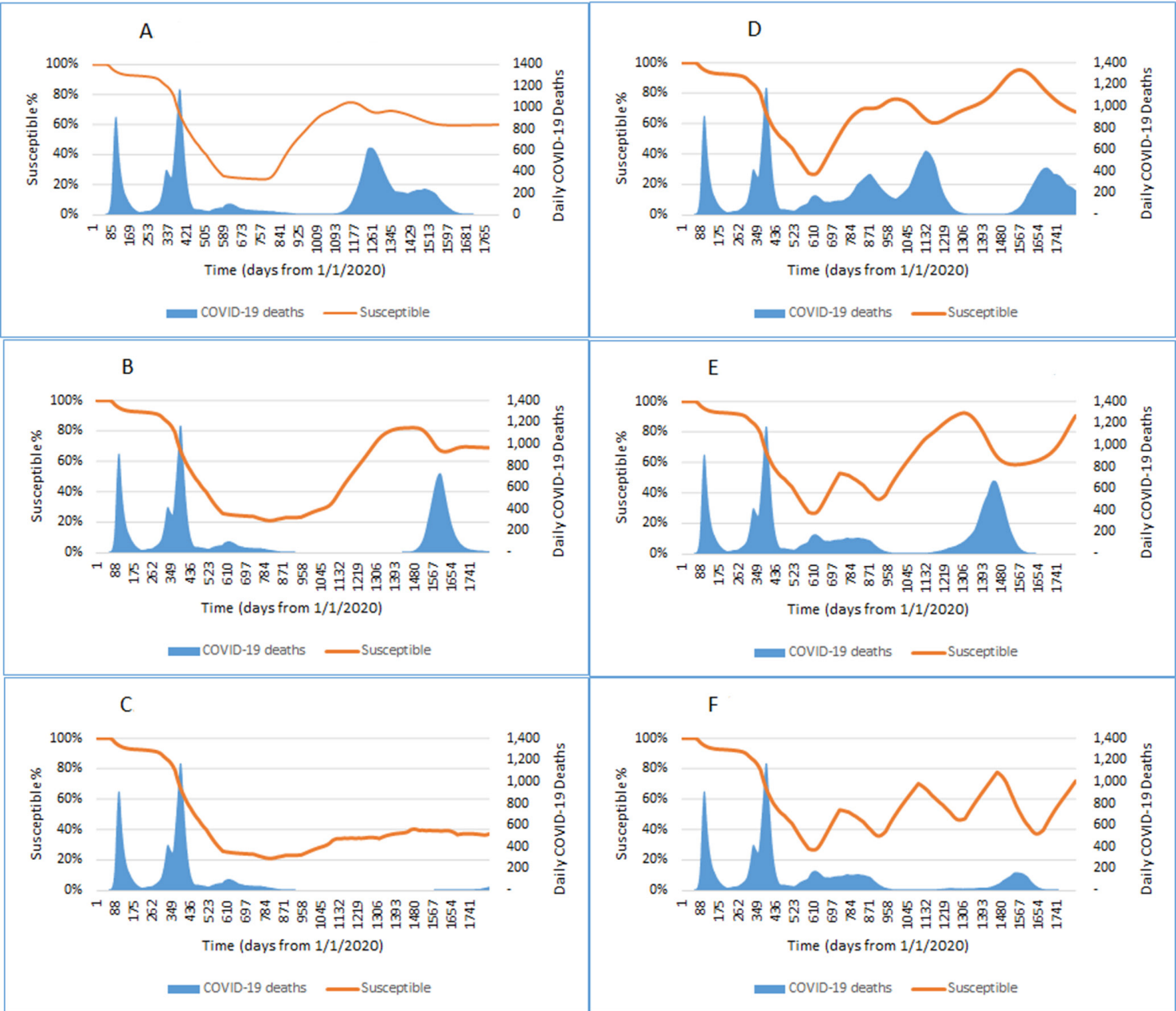

Figure 3 Projected peaks of daily COVID-19 deaths and the prevalence of susceptible individuals during 2020-2024 under scenarios with different immunity durability and vaccine strategies. We assume $85 \%$ long-term vaccine efficacy; $75 \%-$ $90 \%$ coverage of individuals aged $\geq 16 ; 45 \%$ reduction in reinfectivity, longer $(730 / 365$ days, A-C) or shorter lasting (365/182 days, D-F) natural/vaccine immunity. Results were after a single vaccination ( $A$ and $D)$, two annual vaccination programmes ( $B$ and $\mathrm{E}$ ) and four annual vaccination programmes ( $\mathrm{C}$ and $\mathrm{F})$.

\section{Population susceptibility and COVID-19 outbreaks}

Changes in the prevalence of susceptible individuals and daily peaks of COVID-19 deaths in England during 2020 and 2024 are shown in figure 3, under assumptions of $85 \%$ vaccine efficacy after the second dose, $45 \%$ reduction in reinfectivity after waning of sterilising immunity and different durations of sterilising immunity.

The overall prevalence of susceptible individuals is reduced to around 25\% by August 2021, after a single wave of mass vaccination starting from January 2021. It starts to increase to above $70 \%$ by December 2022, if the duration of natural and vaccine-induced immunity is 730 and 365 days, respectively (figure $3 \mathrm{~A}$ ). The raised prevalence of susceptible individuals leads to an outbreak with a high peak $(\mathrm{n}=627)$ of daily COVID-19 deaths in May 2023. The prevalence of the susceptible is reduced from $74 \%$ to $68 \%$ by the natural infection during the outbreak, then slightly increases again $(69 \%)$ due to waning of immunity. After the outbreak wave in January 2024, the prevalence of the susceptible is reduced to around $60 \%$ (figure 3A). Two waves of repeated annual revaccination programmes delay the new outbreak, with a peak of daily COVID-19 deaths $(\mathrm{n}=731)$ in May 2024 (figure 3B). Four repeated waves of annual revaccination programmes almost prevent any new outbreaks before the end of 2024, with no more than 50 daily COVID-19 deaths during 2022-2024 (figure 3C).

If the duration of natural and vaccine immunity lasts only 365 and 182 days, respectively, the annual mass vaccination programmes are insufficient to sustain a constantly low prevalence of the susceptible, and the prevalence of the susceptible fluctuates up and down biannually (figure 3D-F). A single wave of mass vaccination is followed with multiple high peaks of daily COVID-19 deaths (figure 3D), and annual revaccination programmes result in corresponding changes in the prevalence of susceptible individuals and daily COVID-19 deaths (figure 3E,F). With four annual revaccination programmes, there are two flattened peaks of daily deaths from COVID-19 in March $2022(\mathrm{n}=151)$ and April 2024 $(\mathrm{n}=168)$, respectively (figure $3 \mathrm{~F})$. 
Table 1 Projected total and daily peak COVID-19 deaths after annual vaccination programmes by 2024 in England

\begin{tabular}{|c|c|c|c|c|c|c|c|}
\hline Scenario & $\begin{array}{l}\text { Long-term } \\
\text { vaccine efficacy } \\
(\%)\end{array}$ & $\begin{array}{l}\text { Reduction in } \\
\text { reinfectivity (\%) }\end{array}$ & $\begin{array}{l}\text { Immunity } \\
\text { duration (day) } \\
\text { Natural/vaccine }\end{array}$ & $\begin{array}{l}\text { Total COVID-19 } \\
\text { deaths (2020- } \\
2024)\end{array}$ & \multicolumn{3}{|c|}{ Daily peaks of COVID-19 deaths } \\
\hline A1b4 & 85.0 & 60 & $730 / 365$ & 127550 & 28 & 0 & 0 \\
\hline $\mathrm{A} 2 \mathrm{~b} 4$ & 85.0 & 45 & $730 / 365$ & 134421 & 42 & 0 & 36 \\
\hline A3b4 & 85.0 & 30 & $730 / 365$ & 152926 & 64 & 4 & 131 \\
\hline A1c4 & 85.0 & 60 & $365 / 182$ & 148093 & 89 & 2 & 7 \\
\hline $\mathrm{A} 3 \mathrm{c} 4$ & 85.0 & 30 & $365 / 182$ & 229664 & 208 & 145 & 96 \\
\hline B1b4 & 62.5 & 60 & $730 / 365$ & 143233 & 104 & 0 & 0 \\
\hline B2b4 & 62.5 & 45 & $730 / 365$ & 179002 & 209 & 0 & 224 \\
\hline B3b4 & 62.5 & 30 & $730 / 365$ & 233075 & 366 & 3 & 384 \\
\hline
\end{tabular}

Scenario labels are corresponding to those used in online supplemental table 1 . We assume annual vaccination covers $75 \%-90 \%$ of individuals aged $\geq 16$ years; the short-term vaccine efficacy is $62.5 \%$ after the first dose and $85 \%$ after the second dose, and the overall vaccine efficacy is equally attributable to the infection and disease protection. 'Long-term vaccine efficacy' refers to vaccine efficacy in fully vaccinated after January 2022.

\section{Projected COVID-19 deaths under various scenarios}

The projected total numbers and daily peaks of COVID-19 deaths during 2020-2024, under all assumed scenarios, are available in online supplemental tables 1 and 2. If there is no waning of immunity (ie, immunity duration $>4$ years) and high vaccine efficacy, a single vaccination programme may prevent COVID-19 outbreaks during 2022-2024, after returning to normality (online supplemental table 1). Otherwise, repeated vaccination programmes are required to prevent further large outbreaks with high peaks of daily deaths from COVID-19.

Table 1 shows the results of annual vaccination programmes under selected scenarios. With annual revaccinations under optimistic scenarios, the cumulative number of COVID-19 deaths is estimated to be from 130000 to 150000 by the end of 2024. However, the total number of COVID-19 deaths may be up to 431000 by the end of 2024, and the number of daily peak deaths may be as high as around 900 in 2022, 600 in 2023 and 1400 in 2024, under scenarios with compromised long-term vaccine efficacy $(62.5 \%)$, short duration of natural and vaccine immunity $(365 / 182$ days) and small reduction in reinfectivity $(30 \%)$ (table 1$)$.

Results of revaccination programmes with different frequency (from two to six revaccinations during 20212024) are shown in figure 4 and online supplemental table 2. Revaccination programmes every 2 years can prevent outbreaks during 2022-2024 in a scenario with high vaccine efficacy $(85 \%)$, large reduction in reinfectivity $(60 \%)$ and long duration of immunity (730/365 days). Under assumed scenarios, more frequent revaccinations are associated with smaller total numbers and lower peaks of daily deaths from COVID-19 (online supplemental table 2). As expected, frequent revaccinations are particularly important when the immunity response is short lived (figure 4A-D). For example, under scenarios of lower long-term vaccine efficacy $(62.5 \%)$, lower reduction in reinfectivity $(30 \%)$ and shorter duration of immunity $(365 / 182)$, the projected number of total COVID-19 deaths by the end of 2024 is reduced from around 430000 by annual revaccination programmes to around 310000 by more frequent revaccinations every 8 months (online supplemental table 2).

Results of scenarios with lower or higher vaccination coverage in younger adults are presented in online supplemental table 3. As expected, lower coverage of vaccination programmes is associated with larger number of total COVID-19 deaths. For example, the projected cumulative number of COVID-19 deaths by the end of 2024 is around 195000 , under a scenario (labelled as 'A2c4' in online supplemental table 3) of annual revaccination, $85 \%$ vaccine efficacy, short duration of immunity (365/182 days) and $75 \%-90 \%$ vaccination coverage. The number of COVID-19 deaths is reduced to around 183000 in scenario (A2c4y) with $80 \%-90 \%$ coverage, and increased to around 210000 in scenario (A2c4x) with $60 \%-90 \%$ coverage (online supplemental table 3 ).

\section{DISCUSSIONS}

Mass immunisation using efficacious vaccines may enable society safely to return to normality. Repeated vaccination programmes are very likely to be required to prevent further COVID-19 outbreaks, depending on vaccine efficacy, the durability and characteristics of different types of immune response to naturally acquired and vaccineinduced immune responses. Evidence on diseases caused by other common $\mathrm{HCoVs}$ indicated that the infection 


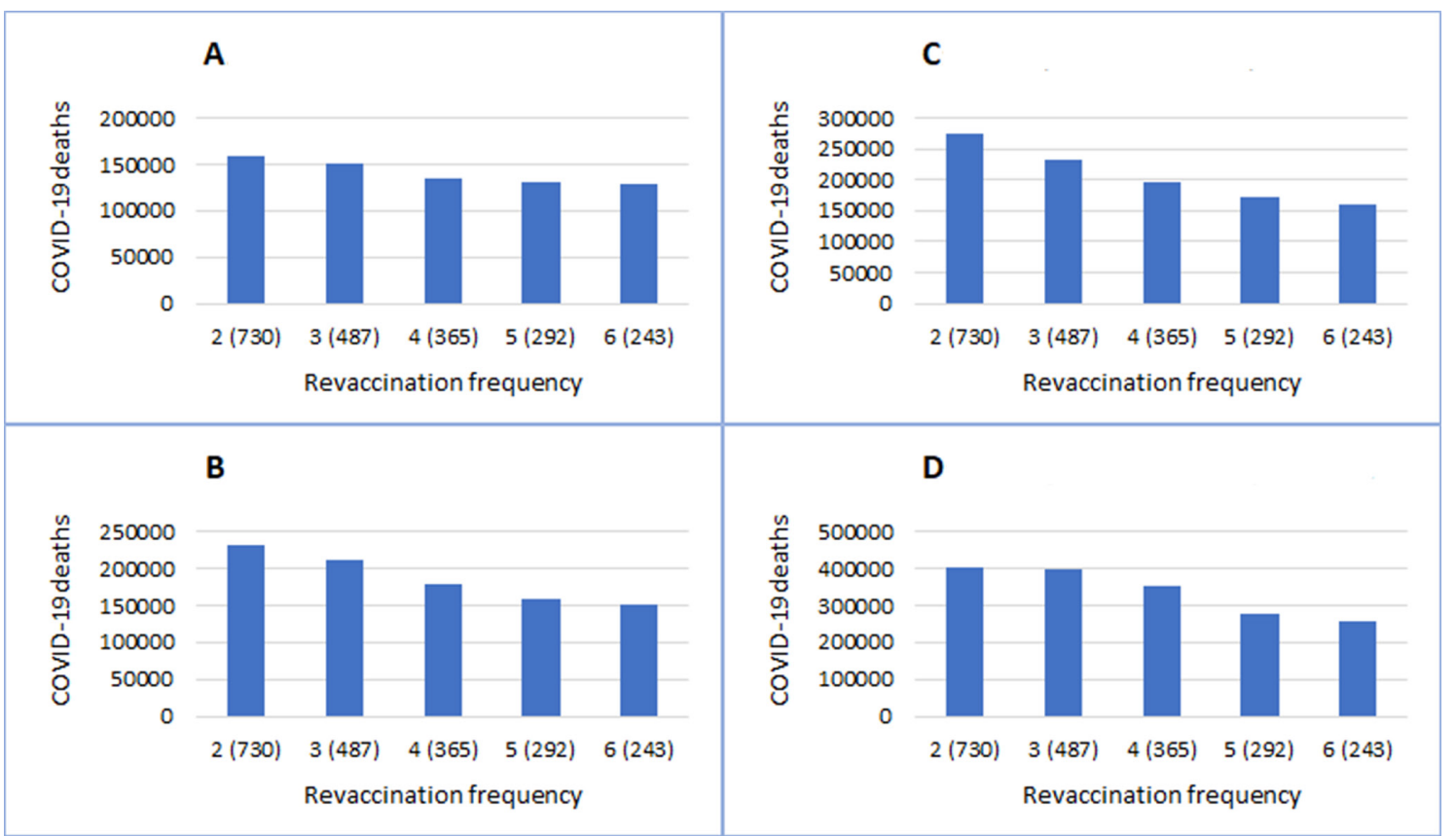

Figure 4 Results of revaccination programmes with different frequency. Vaccine efficacy is $85.0 \%$ in $A / C$ and $62.5 \%$ in $B / D$, respectively. Duration of immunity lasts $730 / 365$ days in $A / B$ and $365 / 182$ days in $C / D$, respectively.

protection immunity may be short lived, but the disease reduction and the reinfectivity reduction immunity are likely to be longer lasting. ${ }^{20}$ We found that, if vaccine efficacy is high $(85 \%)$ and reinfectivity is greatly reduced (eg, by $60 \%)$, repeated annual mass vaccination programmes prevent further COVID-19 large outbreaks, even if the vaccine-induced sterilising immunity lasts only 182 days. Under optimistic scenarios, the cumulative number of COVID-19 deaths during 2020-2024 in England is estimated to be from 130000 to 150000 . If both the natural and vaccine immunity are short lived (365 and 180 days, respectively), and reinfectivity is reduced only by $45 \%$ or $30 \%$, further COVID-19 outbreaks cannot be prevented by annual vaccination programmes. The total number of COVID-19 deaths is estimated to be around 431000 by the end of 2024, under a pessimistic scenario with low long-term vaccine efficacy $(62.5 \%)$, short duration of vaccine immunity $(365 / 182$ days $)$ and small reduction in reinfectivity $(30 \%)$ (table 1$)$.

When natural and vaccine-induced immune response against SARS-CoV-2 infection is short lived, more frequent revaccinations (eg, every 8-10 months) can reduce deaths from COVID-19, compared with less frequent or annual revaccination programmes. However, frequent revaccination may not always be feasible due to the availability of vaccine doses, resource restrictions, organisational complexity and possibly decreased compliance by the public.

Compared with previous modelling studies, ${ }^{14-17}$ our study has considered the durability of naturally acquired and vaccine-induced immunity, over a 5-year period from 2020 to 2024, and compared a wider range of plausible scenarios. We explicitly investigated the impacts of different types of immune responses to SARS-CoV-2 infection and vaccines on the COVID-19 epidemic in a country. Findings from our study will improve the understanding of key immunological parameters relevant to future changes in SARS-CoV-2 transmission dynamics and vaccination strategies.

Evidence from randomised controlled trials showed that vaccines against SARS-CoV-2 are efficacious in reducing symptomatic COVID-19 cases. ${ }^{5}$ The reduction in symptomatic cases in the vaccine group may be attributable to infection protection or disease reduction. Available evidence showed that vaccines reduced the risk of SARS-CoV-2 infection in vaccinated individuals, ${ }^{38}$ and household members of vaccinated healthcare workers have a lower risk of COVID-19 infection than those of unvaccinated. ${ }^{39}$ In this study, we explored the impacts of different proportions of a vaccine's infection protection efficacy and disease reduction efficacy. Different combinations of the two efficacy components have impacts on the transmission dynamics, depending on the duration of immune response and reinfectivity after waning of sterilising immunity. Because of lack of data, we assumed that vaccine efficacy is equally attributable to the infection and disease reduction immunity.

Vaccines that are efficacious against current SARSCoV-2 may become less efficacious against new emerging variants. ${ }^{9}$ Therefore, we modelled a range of overall longterm vaccine efficacy $(62.5 \%$ vs $85.0 \%)$. Although the vaccine efficacy might be reduced by new viral variants, it is also possible that repeated vaccinations may boost immune responses. ${ }^{40}$ We assume that there will be no important safety issues for vaccines licensed to use. We focused on the impacts and interactions of vaccine efficacy, different types of immune response to SARS-CoV-2, and assumed no more restrictions by NPI measures after 
return to normality in England on 19 July 2021. However, the pessimistic scenarios in our analyses may not be allowed to happen in the real world, as NPI (including lockdown) measures may have to be introduced again if the vaccination programmes are insufficient to avoid the new outbreaks of COVID-19.

This is a deterministic simulation model, and uncertainty in estimated parameters may have not been fully accommodated. For simplicity, stochastic uncertainty, to quantify CIs around the model's outputs, was not modelled. However, the model has been calibrated based on historically observed outcome data in England, and a large number of projection scenarios are explored to explicitly answer 'what-if' questions. Although many uncertainties remain, including durability and types of naturally acquired and vaccine-induced immunity, our model can be updated to assess vaccination strategies, as new evidence emerges.

\section{CONCLUSIONS}

Under optimistic scenarios, mass immunisation using efficacious vaccines may enable society safely to return to normality. However, under plausible scenarios with low vaccine efficacy and short durability of immunity, COVID-19 could continue to cause recurrent waves of severe morbidity and mortality despite frequent vaccinations, and necessitate stringent NPI restrictions. It is crucial to monitor the vaccination effects in the real world, and to better understand the characteristics of naturally acquired and vaccine-induced immunity against SARS-CoV-2.

Acknowledgements We used UEA high-performance computing cluster for this study, and received support from the Research and Specialist Computing Service, University of East Anglia.

Contributors FS designed and developed the model, retrieved data for estimating parameters, conducted computational calculations and prepared the draft manuscript. MOB provided methodological support, helped interpret results and critically revised the draft manuscript. FS is the guarantor and accepts full responsibility for the work and/or the conduct of the study, had access to the data and controlled the decision to publish.

Funding The authors have not declared a specific grant for this research from any funding agency in the public, commercial or not-for-profit sectors.

Competing interests None declared.

Patient consent for publication Not applicable.

Provenance and peer review Not commissioned; externally peer reviewed.

Data availability statement Data are available upon reasonable request.

Supplemental material This content has been supplied by the author(s). It has not been vetted by BMJ Publishing Group Limited (BMJ) and may not have been peer-reviewed. Any opinions or recommendations discussed are solely those of the author(s) and are not endorsed by BMJ. BMJ disclaims all liability and responsibility arising from any reliance placed on the content. Where the content includes any translated material, BMJ does not warrant the accuracy and reliability of the translations (including but not limited to local regulations, clinical guidelines, terminology, drug names and drug dosages), and is not responsible for any error and/or omissions arising from translation and adaptation or otherwise.

Open access This is an open access article distributed in accordance with the Creative Commons Attribution Non Commercial (CC BY-NC 4.0) license, which permits others to distribute, remix, adapt, build upon this work non-commercially, and license their derivative works on different terms, provided the original work is properly cited, appropriate credit is given, any changes made indicated, and the use is non-commercial. See: http://creativecommons.org/licenses/by-nc/4.0/.

\section{ORCID iDs}

Fujian Song http://orcid.org/0000-0002-4039-1531

Max 0 Bachmann http://orcid.org/0000-0003-1770-3506

\section{REFERENCES}

1 The World Health Organization. WHO coronavirus (COVID-19) Dashboard, 2021. Available: https://covid19.who.int/ [Accessed 10 Aug 2021].

2 Islam N, Sharp SJ, Chowell G, et al. Physical distancing interventions and incidence of coronavirus disease 2019: natural experiment in 149 countries. BMJ 2020;370:m2743.

3 Krammer F. SARS-CoV-2 vaccines in development. Nature 2020;586:516-27.

4 Department of Health and Social Care. Optimising the covid-19 vaccination programme for maximum short-term impact, 2021. Available: https://www.gov.uk/government/publications/prioritisingthe-first-covid-19-vaccine-dose-jcvi-statement/optimising-the-covid19-vaccination-programme-for-maximum-short-term-impact

5 Mallapaty S. Can COVID vaccines stop transmission? Scientists race to find answers. Nature 2021. doi:10.1038/d41586-021-00450-z. [Epub ahead of print: 19 Feb 2021].

6 Bingham K. The UK government's vaccine Taskforce: strategy for protecting the UK and the world. Lancet 2021;397:68-70.

7 GOV.UK. The official UK government website for data and insights on coronavirus (COVID-19), 2021. Available: https://coronavirus.data. gov.uk/ [Accessed 10 Aug 2021].

8 Gov.uk. Coronavirus (COVID-19): guidance and support, 2021. Available: https://www.gov.uk/coronavirus [Accessed 30 Jul 2021]

9 Mahase E. COVID-19: where are we on vaccines and variants? BMJ 2021:372:n597.

10 Sanyaolu A, Okorie C, Marinkovic A, et al. The emerging SARS-CoV-2 variants of concern. Ther Adv Infect Dis 2021;8:20499361211024372.

11 Ferguson N, Laydon D, Nedjati-Gilani G. Impact of nonpharmaceutical interventions (NPIs) to reduce COVID-19 mortality and healthcare demand. Imperial College COVID-19 Response Team, 2020.

12 Davies NG, Kucharski AJ, Eggo RM, et al. Effects of nonpharmaceutical interventions on COVID-19 cases, deaths, and demand for hospital services in the UK: a modelling study. Lancet Public Health 2020;5:e375-85.

13 Giordano G, Blanchini F, Bruno R, et al. Modelling the COVID-19 epidemic and implementation of population-wide interventions in Italy. Nat Med 2020;26:855-60.

14 Cook TM, Roberts JV. Impact of vaccination by priority group on UK deaths, hospital admissions and intensive care admissions from COVID-19. Anaesthesia 2021;76:608-16.

15 Miles DK, Heald AH, Stedman M. How fast should social restrictions be eased in England as COVID-19 vaccinations are rolled out? Int $J$ Clin Pract 2021;75:e14191.

16 Moore S, Hill EM, Tildesley MJ. Vaccination and non-pharmaceutical interventions for COVID-19: a mathematical modelling study. Lancet Infect Dis 2021 (published Online First: 2021/03/22).

17 Sandmann FG, Davies NG, Vassall A, et al. The potential health and economic value of SARS-CoV-2 vaccination alongside physical distancing in the UK: a transmission model-based future scenario analysis and economic evaluation. Lancet Infect Dis 2021;21:962-74.

$18 \mathrm{Li} \mathrm{J}$, Giabbanelli P. Returning to a normal life via COVID-19 vaccines in the United States: a large-scale Agent-Based simulation study. JMIR Med Inform 2021;9:e27419.

19 Hodgson SH, Mansatta K, Mallett G, et al. What defines an efficacious COVID-19 vaccine? A review of the challenges assessing the clinical efficacy of vaccines against SARS-CoV-2. Lancet Infect Dis 2021;21:e26-35.

20 Lavine JS, Bjornstad ON, Antia R. Immunological characteristics govern the transition of COVID-19 to endemicity. Science 2021;371:741-5.

21 R Core Team. R: a language and environment for statistical computing. Vienna, Austria: R Foundation for Statistical Computing, 2019. https://www.R-project.org/

22 GOV.UK. Coronavirus (COVID-19) in the UK, 2021. Available: https:// coronavirus.data.gov.uk/ [Accessed 31 Jul 2021].

23 Office for National Statistics. Dataset: 2018-based National population projections table of contents, 2018. 
24 Zhang J, Litvinova M, Wang W, et al. Evolving epidemiology and transmission dynamics of coronavirus disease 2019 outside Hubei Province, China: a descriptive and modelling study. Lancet Infect Dis 2020;20:793-802.

25 Verity R, Okell LC, Dorigatti I. Estimates of the severity of coronavirus disease 2019: a model-based analysis. Lancet Infect Dis 2020.

26 Rock K, Brand S, Moir J, et al. Dynamics of infectious diseases. Rep Prog Phys 2014;77:026602.

27 Mahase E. Covid-19: what have we learnt about the new variant in the UK? BMJ 2020;371:m4944.

28 Rubin D, Huang J, Fisher BT, et al. Association of social distancing, population density, and temperature with the instantaneous reproduction number of SARS-CoV-2 in counties across the United States. JAMA Netw Open 2020;3:e2016099.

29 Mossong J, Hens N, Jit M, et al. Social contacts and mixing patterns relevant to the spread of infectious diseases. PLoS Med 2008;5:e74.

30 Mahase E. Covid-19: UK approves pfizer and BioNTech vaccine with rollout due to start next week. BMJ 2020;371:m4714.

31 Department of Health \& Social Care JCVI statement,. August 2021: COVID-19 vaccination of children and young people aged 12 to 17 years.: Department of Health \& Social Care, 2021. Available: https:// www.gov.uk/government/organisations/department-of-health-andsocial-care

32 PHE. COVID-19 vaccine surveillance report week 28: public health England, 2021.
33 Le Bert N, Tan AT, Kunasegaran K, et al. SARS-CoV-2-specific T cell immunity in cases of COVID-19 and SARS, and uninfected controls. Nature 2020;584:457-62.

34 Levine-Tiefenbrun M, Yelin I, Katz R. Decreased SARS-CoV-2 viral load following vaccination. : . medRxiv 2021:2021.02.06.21251283. \%J.

35 Emary KRW, Golubchik T, Aley PK. Efficacy of ChAdOx1 nCoV-19 (AZD1222) vaccine against SARS-CoV-2 VOC 202012/01 (B.1.1.7). SSRN [Preprint] 2021.

36 Mallapaty S. COVID vaccines slash viral spread - but Delta is an unknown. Nature 2021;596:17-18.

37 Government Office for Science and Scientific Advisory Group for Emergencies. Guidance: the R number and growth rate in the UK from government office for science and scientific Advisory group for emergencies., 2020. Available: https://www.gov.uk/guidance/the-rnumber-in-the-uk\#contents [Accessed 08 Oct 2020].

38 Hall VJ, Foulkes S, Charlett A, et al. SARS-CoV-2 infection rates of antibody-positive compared with antibody-negative health-care workers in England: a large, multicentre, prospective cohort study (siren). Lancet 2021;397:1459-69.

39 Shah AS, Gribben C, Bishop J. Effect of vaccination on transmission of COVID-19: an observational study in healthcare workers and their households. medRxiv 2021:2021.03.11.21253275.

40 Callaway E. COVID vaccine boosters: the most important questions. Nature 2021;596:178-80. 